\title{
Corf-940/42--25
}

UCRL-JC-115842

PREPRINT

\section{Microanalytical Study of Defect Formation in Thin Bismuth Strontium Calcium Copper Oxide Films}

\author{
R.H. Howell, A. Chaiken, R.G. Musket, M.A Wall \\ M. Balooch, D. Phinney and M.J. Fluss \\ Lawrence Livermore National Lab \\ Livermore, CA 94550
}

J.N. Eckstein, I. Bozovic, and G.F. Virshup

Ginzton Research Center, Varian Associates

Palo Alto, CA

Prepared for submittal to

OE/LASE '94, Los Angeles, CA

January 22-29, 1994

January 1994

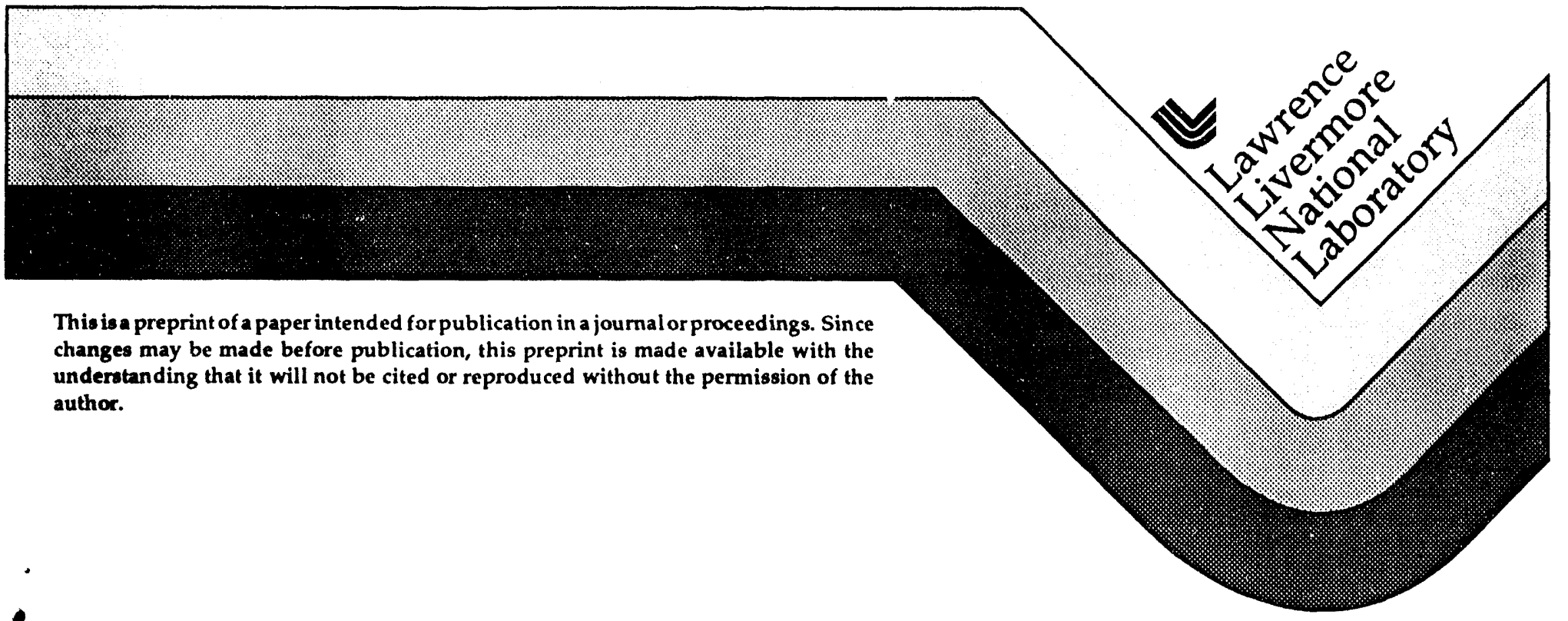

\section{MASTER}

DISTRIBUTION OF THIS DOCUMENT IS UNLIMITED 


\section{DISCLAIMER}

This document was prepared as an account of work sponsored by an agency of the United States Government. Neither the United States Government nor the University of California nor any of their employees, makes any warranty, express or implied, or assumes any legal liability or responsibility for the accuracy, completeness, or usefulness of any information, apparatus, product, or process disclosed, or represents that its use would not infringe privately owned rights. Reference herein to any specific commercial products, process, or service by trade name, trademark, manufacturer, or otherwise, does not necessarily constitute or imply its endorsement, recommendation, or favoring by the United States Government or the University of California. The views and opinions of authors expressed herein do not necessarily state or reflect those of the United States Government or the University of California, and shall not be used for advertising or product endorsement purposes. 


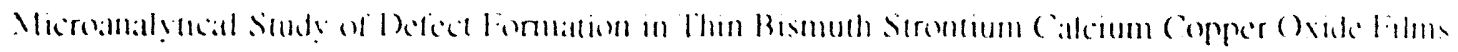

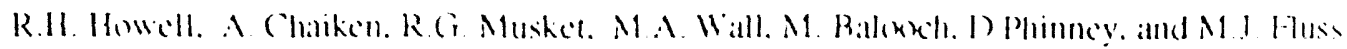

1.awrence livermore Nationall lah. I ivermmore ('A

IN. Eckstein. I. Bozovic. and (i l. Virshup

Ginzton Rescauch Center, Varian Assckiales. Palo Allo, CA.

\begin{abstract}
Thin bismuth strontium calcium copper oxide (BSCCO) films and BSCCO/insulator/BSCCO trilavers have been prepared on $\mathrm{SrTiO}_{3}$ and $\mathrm{MgO}$ substrates by evaporation from elemental sources in an ozone atmosphere. Accurate control of the stoichiometry is achieved through monitoring of the atomic fluxes by use of in sitn atomic absorption sp sctroscopy. as well as by reflection high-energy electron diffraction (RHEED). Nevertheless. nanometer-scale second-phase precipitates are sometimes observed. These defects and the that regions around them have been probed by a variety of microanalytical techniques. including Rudertord backscattering spectroscopy (RBSS), particle-induced $x$-ray emission (PIXE). atomic force microscopy (AFM). microscopic secondary ion mass spectroscopy and trinsmissinn electron mictoscopy (TEM).
\end{abstract}

\title{
INTRODUCTION
}

The ideal properties of high temperature superconducting oxide materials make them appropriate for many applications in both high current and electronic applications. These include current-carrying tapes for high-field electromagnets, electronic devices for microwave applications. and tumel junction devices for sensors and electronics. Applications of films of high temperature superconductors require varying degrees of approximation to ideal characteristics. Current-carrying films require some limitations on texture. Films to make SQUDs for sensors must be well-oriented but can wherate significant levels of local defects. However, films for some tynes of electronic devices must approach the order of perfection of macroscopic single-crystalline structures. Films with such a high level of order can be fabricated by epitaxial deposition of the superconducting material onto an appropriate substrate witi strict controls on the deposition rates and environment.

In this paper we report on attempts to better understand the details of the deposition process. the resulting microstructure and device characteristics. We will demonstrate the high level of order of films deposited by high-vacuum elaporation at Varian. Preliminary observations about the most common defects will be discussed. In the Varian Reactive Chemical Epitaxy (RCE) process fine control over the deposition rate is achieved by in situ RHEED monitoring of the film surtace during deposition [1-4], supplemented by atomic adsorption measurements of the flux.[5] Material is deposited in molecular lavers so that atomistic control of the composition of each unit cell is achieved and complex structures can be formed. This procedure results in films of high quality over significant portions of the deposition areat. When grown as multilayer composites, these films can be pattemed into high-quahty Josephson junctions with c-atis transport 6 ].

\section{Film qualit:}

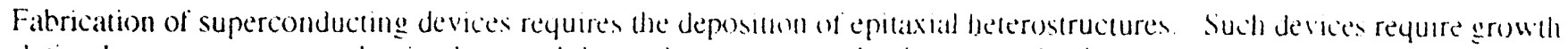
of an insulating haver on a superconducting base and then subsequent grow th of superconducting overlaters with good epitaxial

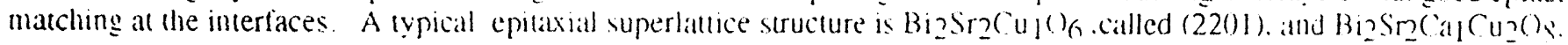

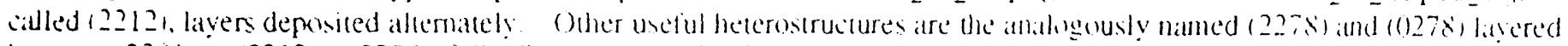

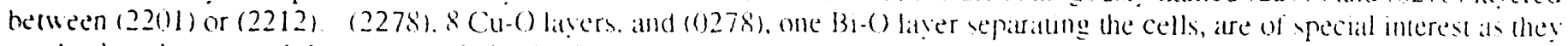

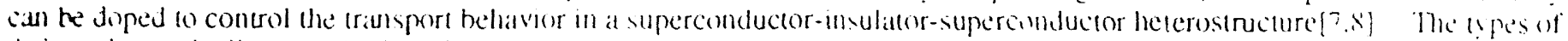
defect that typically occur in these heterostructures include problem. with matching all the interfaces berween differem

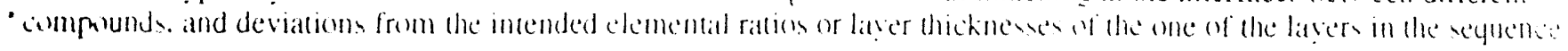




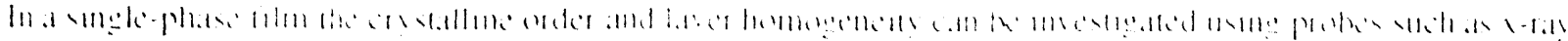

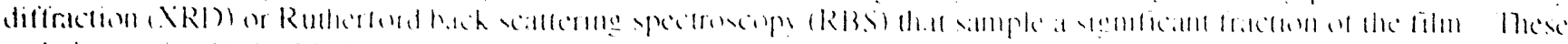

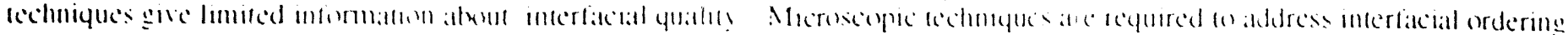

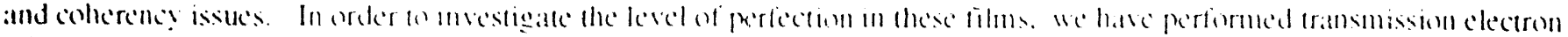

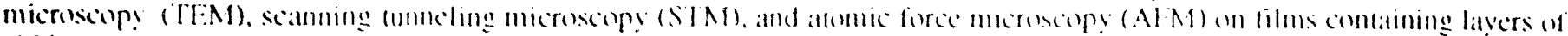

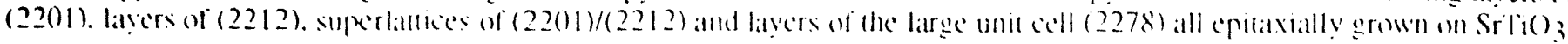

TEM images were whtamed in a view transverse to the film surface so that rach of the separate lavers could be individually seen. Plan-view images were ohtained on the surface of the film with AFM and SlM Samples lor AFM and STM are measured as received. For TFM destructive sample preparation was required. Samples were thimed by mechanical polishinge

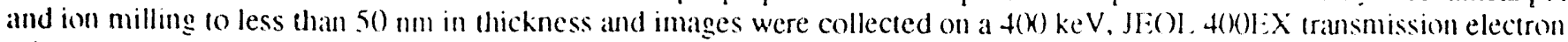
microsiones.

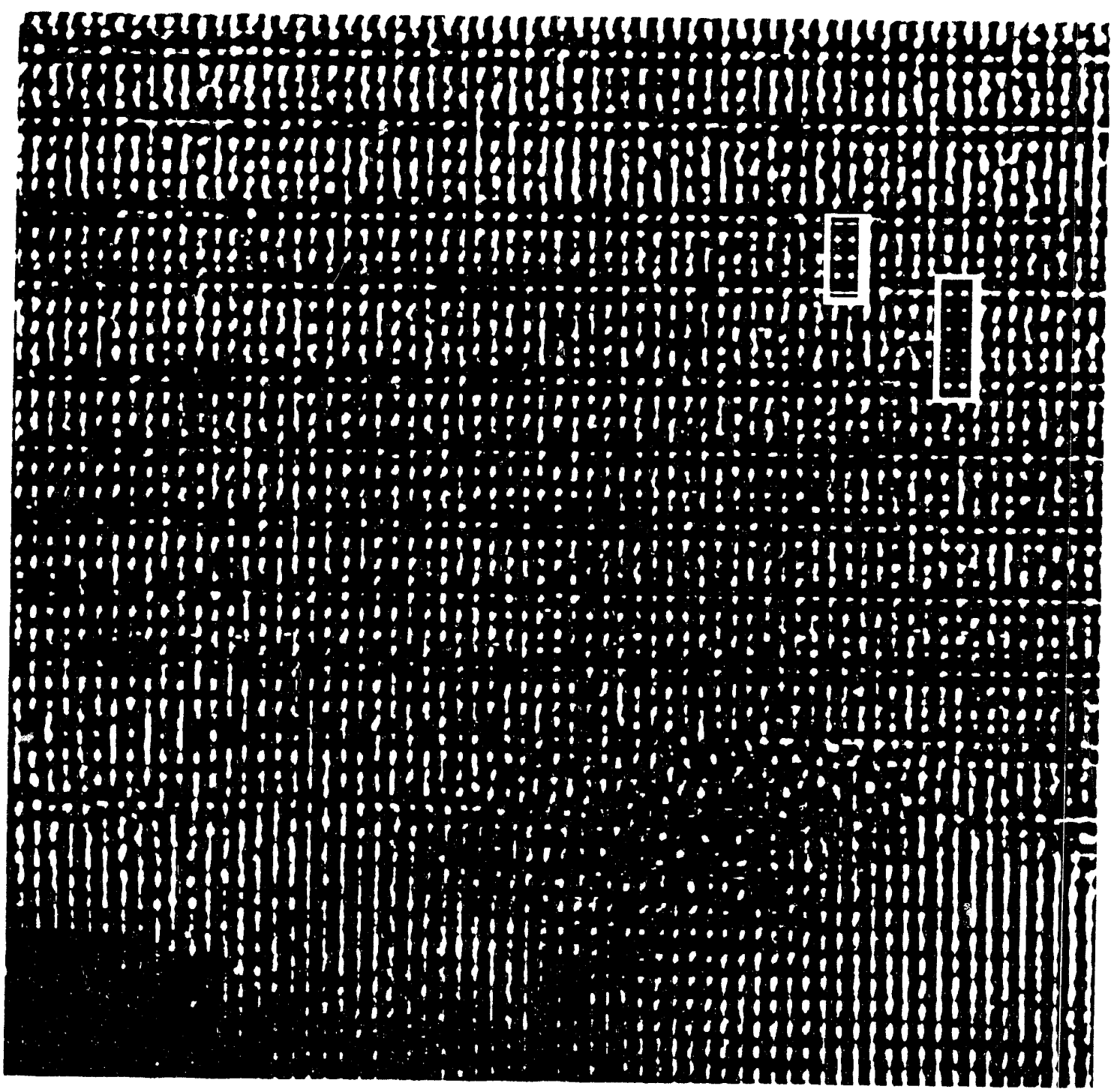

Figure 1

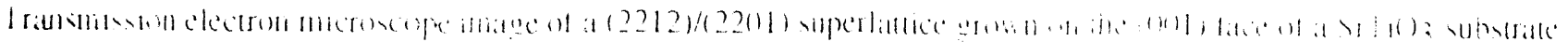




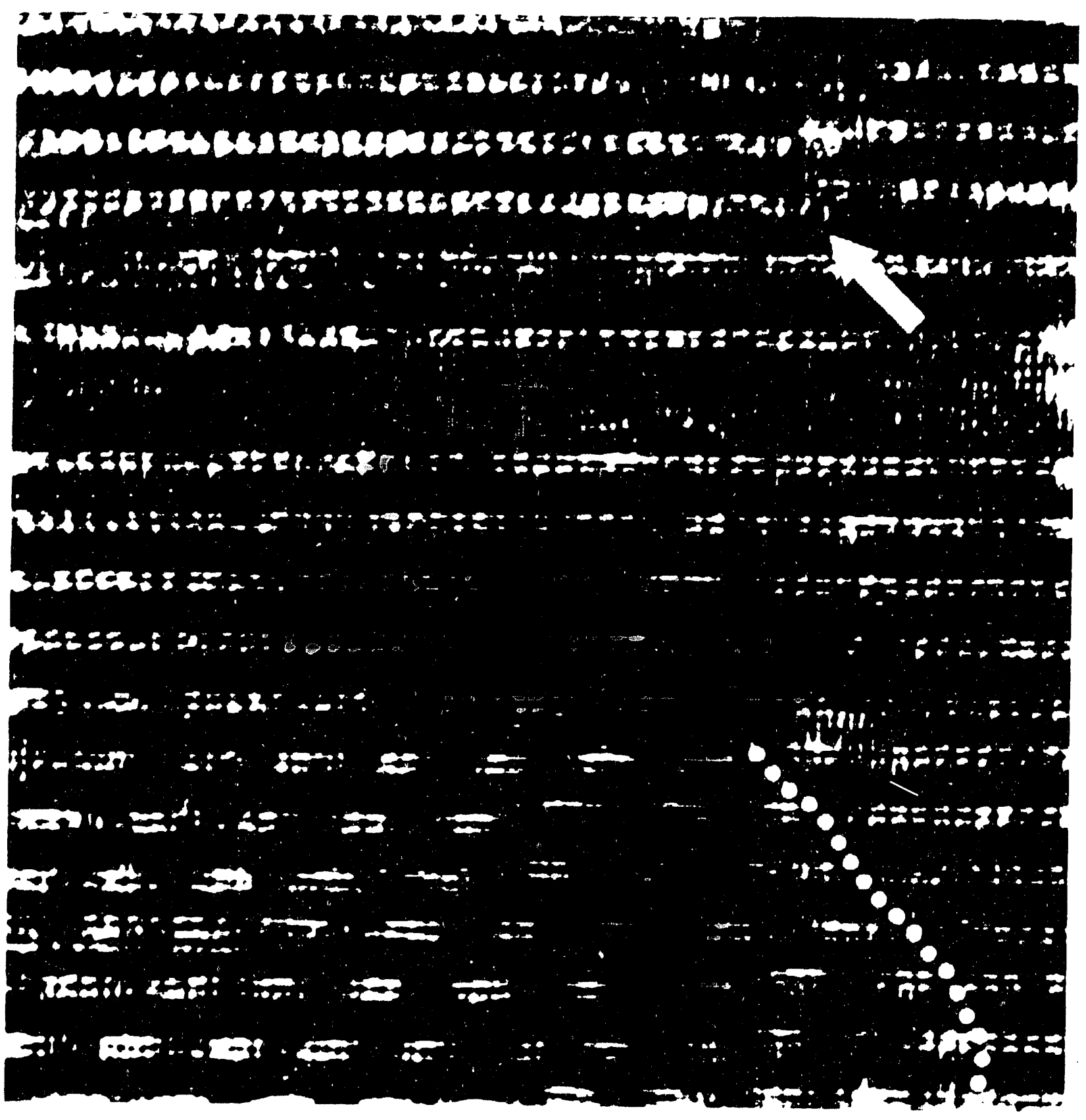

Higure?

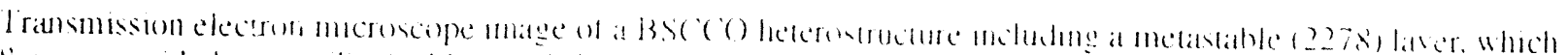

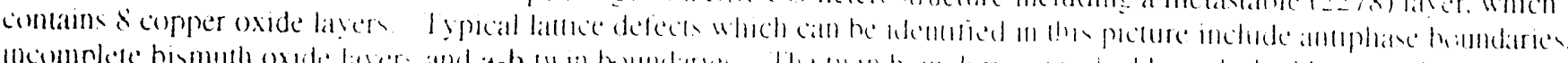

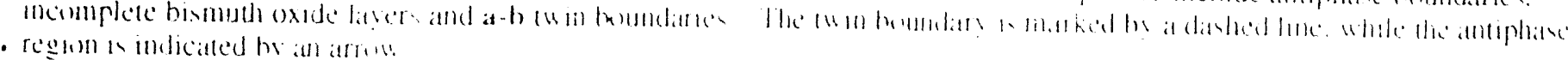




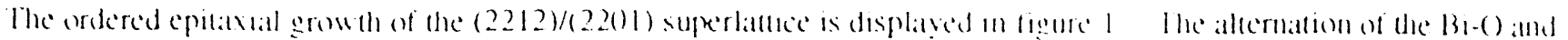
(u-O latyers in the unit cell and the variation in the $1.54 \mathrm{~nm}$ layer spacing of (2212) and ilke 1.22 nm latyer spacing of (2201) is evident. Areas enclosed by the boxes are calculated TFM images for (2201) and (2212). Several features lypical of successtul film grow'th are evident in this picture. The surface roughness of the Srlio) 3 substrate is goked on a microscopic length scale and the furs BSCCO layers have gexel coherence and epitaxial matching. The epitaxy spans the imperfections in the substrate seen midway in the frame. There is a strong tendency for the 13 SCCO system to match the exposed substrate lattice and to construct single continuous layers as the film thickness increases. The crystalline perfection of the (2201)/(2212) superlattice demonstrates the delicate control over deposition that can be obtained in the BSCCO system using RCE.

A second, more dramatic example of heteroepitaxy is seen in figure 2. In this case the intended structure is (2212) $/(2278) /(2212)$. This structure is the basis for fabrication of Josephson junctions with novel. controlled properties. The tunneling characteristics of junctions with c-axis transport fabricated from this and closely related trilayer structures have been reported in [6]. Good boundary matching is seen at the interface of the barrier layer and the superconductor, but there are several imperfections visible in the figure 2. Above the barrier layer there is a break in the continuity of the double BiO layer separating two (2212) layers and an anti-phase boundary defect indicated by the arrow. At the break in the Bi-O layers is the abutment of a new set of phases with the layer spacing of (2223) and (3312), i.e. an extra Bi-O layer on one side and an extra $\mathrm{Cu}-\mathrm{O}$ layer on the other. The anti phase boundary in nearby layers extends over several unit cells for which the continuity of the $\mathrm{Bi}-\mathrm{O}$ layers is completely interrupted. Below the barrier layer there is a twin boundary separating regions of orientation of the a and $\mathbf{b}$ axes as indicated by the dotted line and within the minority twin region is a second anti-phase boundary.

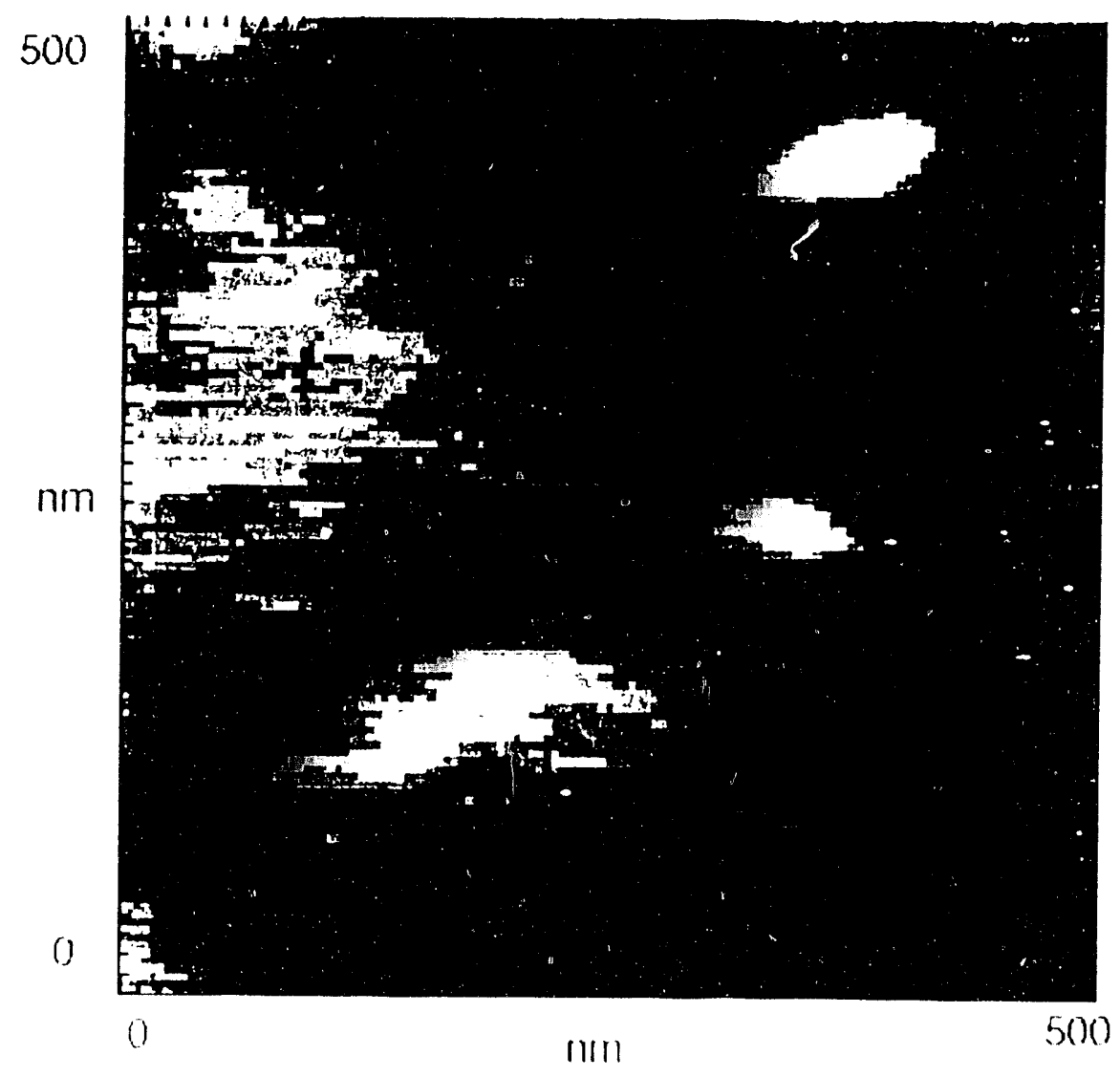

Figure 3

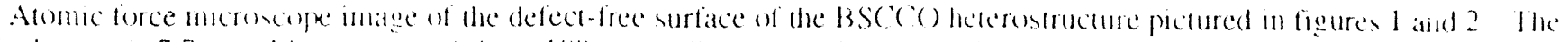

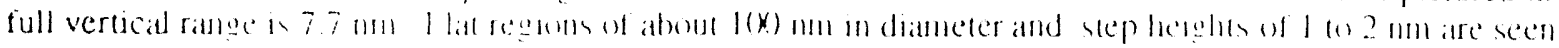




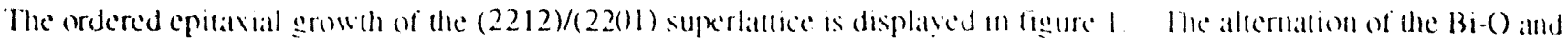
Cu-() layers in the unit cell and the variation in the $1.54 \mathrm{~mm}$ layer spacing of $(2212)$ and the 1.23 mm layer spacing of (2201) is evident. Areas enclosed by the toxes are calculated TEM images for (2201) and (2212). Several leatures typical of successtul film growth are evident in this picture. The surface roughness of the SrliO) 3 substrate is goxd on at microscopic length scale and the furst BSCCO layers have goxt coherence and epitaxial matching. The epitaxy spans the imperfections in the substrate seen miduay in the frame. There is a strong tendency for the BSCCO system to match the exposed substrate lattice and to construct single continuous layers as the film thickness incteases. The crystalline perfection of the (2201)/(2212) superlattice demonstrates the delicate control over deposition that can be obtained in the BSCCO system using RCE.

A second, more dramatic example of heteroepitaxy is seen in figure 2. In this case the intended structure is (2212) /(2278)/(2212). This structure is the basis for fabrication of Josephson junctions with novel. controlled properties. The tunneling characteristics of junctions with c-axis transport fabricated from this and closely related trilayer structures have been reported in [6]. Good boundary matching is seen at the interface of the barrier layer and the superconductor, but there are several imperfections visible in the figure 2. Above the barrier layer there is a break in the continuity of the double BiO layer separating two (2212) layers and an anti-phase boundary defect indicated by the arrow. At the break in the Bi-O layers is the abutment of a new set of phases with the layer spacing of (2223) and (3312), i.e. an extra Bi-O layer on one side and an extra $\mathrm{Cu}-\mathrm{O}$ layer on the other. The anti phase boundary in nearby layers extends over several unit cells for which the continuity of the $\mathrm{Bi}-\mathrm{O}$ layers is completely interrupted. Below the barrier layer there is a twin boundary separating regions of orientation of the $\mathbf{a}$ and $\mathbf{b}$ axes as indicated by the dotted line and within the minority twin region is a second anti-phase boundary.

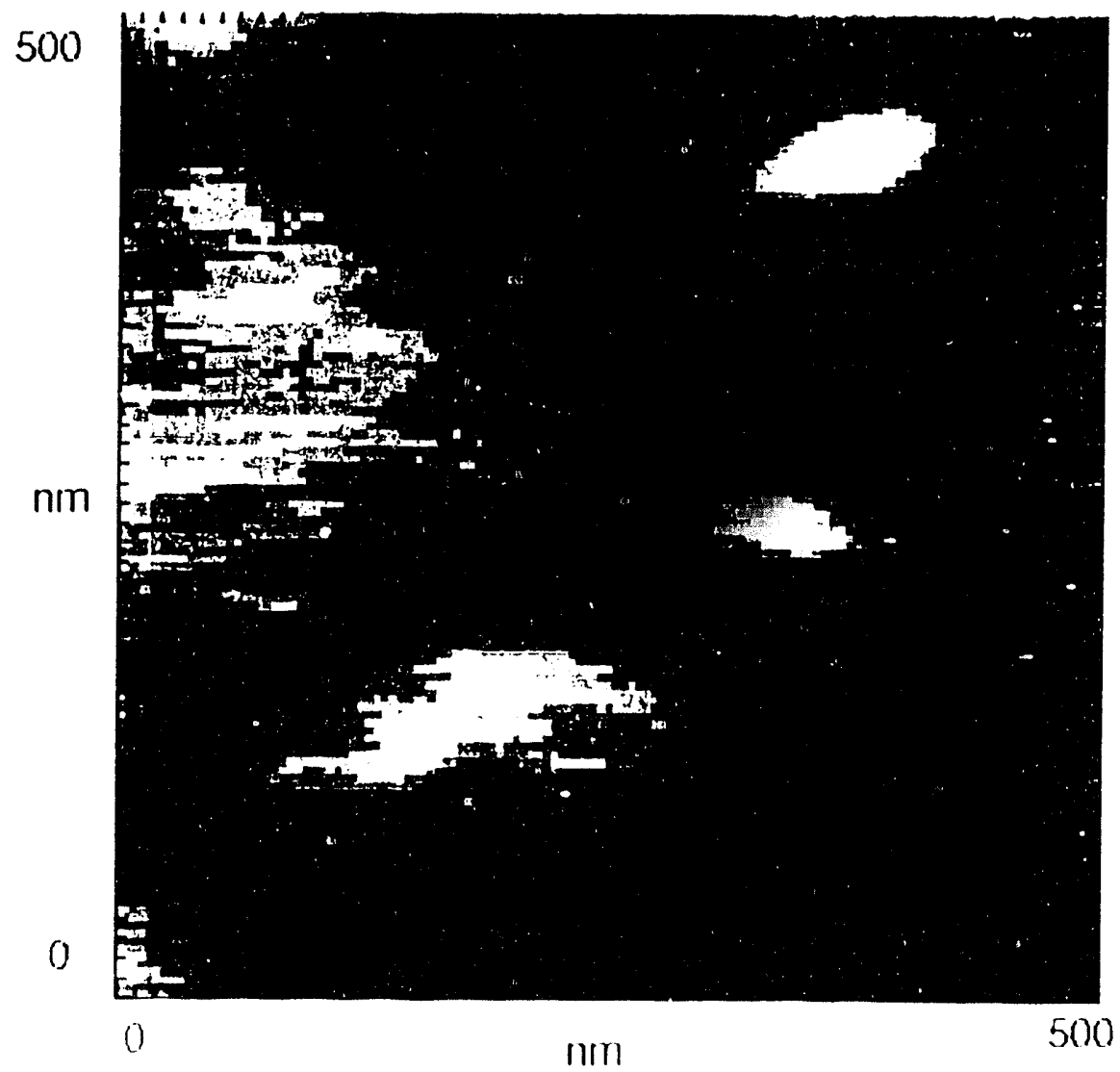

Figure 3

Atomic force microscope image of the defect-free surtace of the BSCC(O) helerostructure pictured in ligures 1 and 2 . The

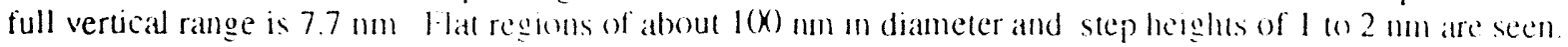




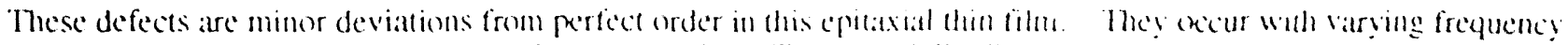
throughout the volume of the films that have so far been studied. The spatial distribution of the interruptions to continuity in the Bi-O planes can be determined from examination of TTM images taken from several parts of the film. The scale of the roughness in the surface of the film can be independently determined by AFM. An AFM image of the smooth surface of the sume film as used to ohtain the IFM images is seen in figure 3. In this image we sec surfice roughiness with a lateral scale of roughly $1(K) \mathrm{nm}$ and a vertical scale of $7.7 \mathrm{~nm}$. The roughness is not randomly distributed but appears in steps having a typical step size of about $1.5 \mathrm{~mm}$. This corresponds closely with the $\mathrm{c}$ ax is layer spacing of $1.54 \mathrm{~mm}$ for the terminating Bi(2\%12) phase. This is a surface with a high degree of snoothness. In some parts of the sample there are surface asperities distrituted with a spacing of microns or larger. The roughness variations in the vertical direction in figure 3 are typical of those found in defect-free regions on all of the samples measured and the scale of the in plane variations are consistent with those taken from TEM.

Microscopic roughness in the substrate surface is acconmodated by the strong tendency toward long in-plane colnerence in BSCCO. However, large-scale substrate roughness can serve to initiate breaks in layers. For instance, in the $\mathrm{YBa}_{2} \mathrm{Cu}_{3} \mathrm{O}_{7}$ system edge junctions are formed at high steps patterned in the substrates. We have investigated the roughness of randomly selected $\mathrm{SrTiO}_{3}$ substrates. Both optical intefferometery and AFM determinations of the substrate surface were performed. In optical interferometry the root-mean-square of the substrate roughness was typically $0.2 \mathrm{~nm}$. In AFM similar roughness was observed. With both techniques the BSCCO film surface was found to be at least as smooth as the substrate in most regions.

A low density of larger size defects can be observed on the surface of nearly all films made by the RCE process. In good films these features only have a significant density at the edges of the substrate water. However. in tilms deposited under nonoptimal conditions bigh densities of defects have been observed on the surface. The largest of these have micron scale dimensions and can be seen in an optical microscope. A high density of these surface defects is correlated with degraded Iosephson junction performance. At present the source and composition of these defects is not well understood. There may be several defect types resulting from separate initiation processes in the film deposition.

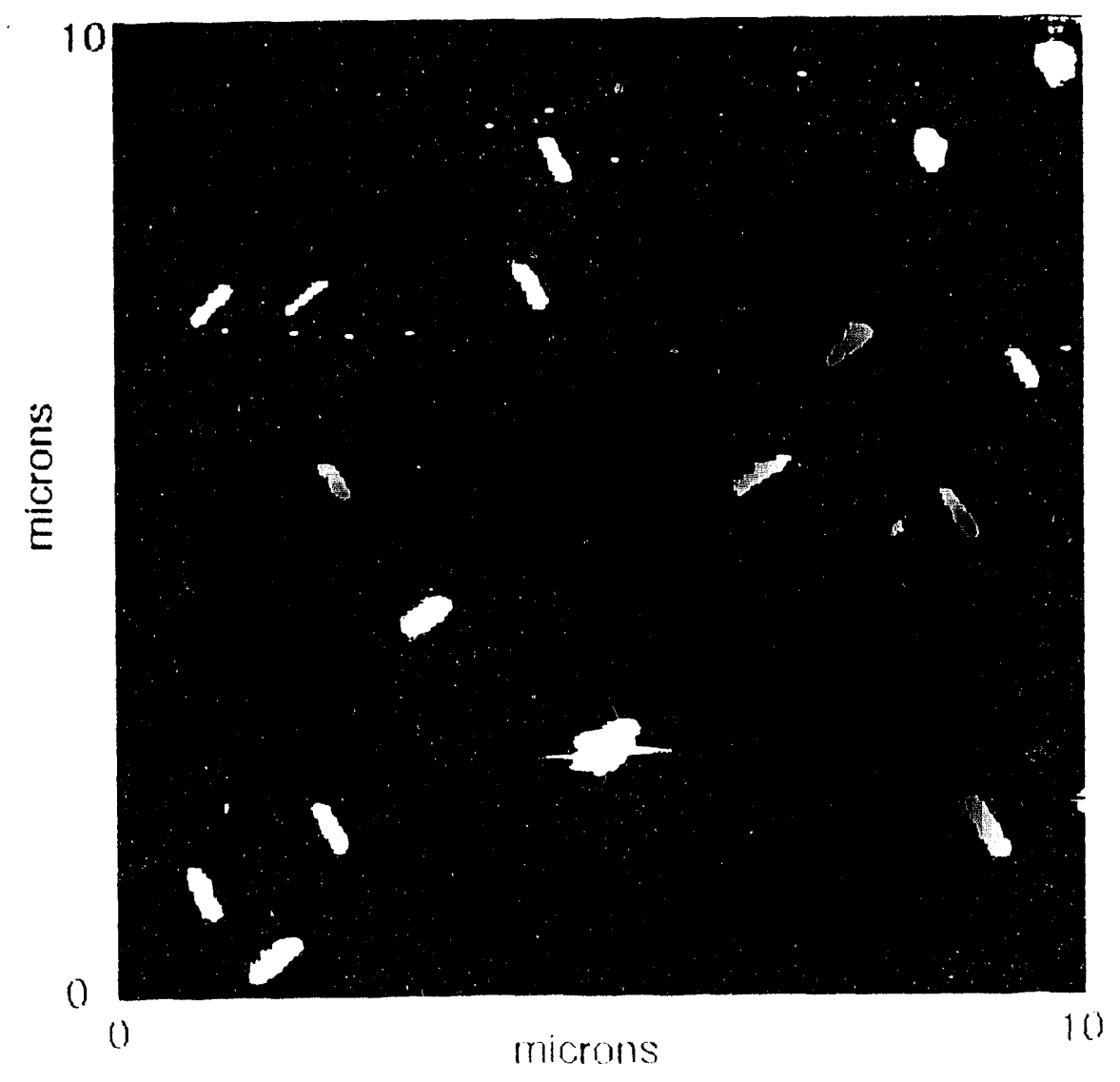

Figure 4

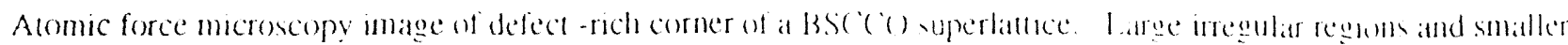
oriented defeces buth protrude from the smmenth sample surlate 


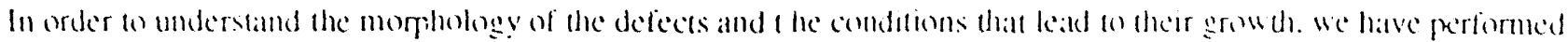

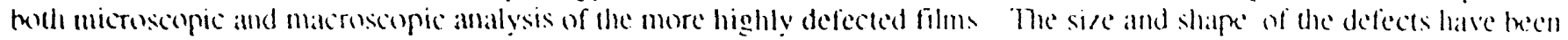
imaged using hoh AFM and STM. Microscopic images of elemental composition in a defected region have been imaged using an ion microscopx. While dle macroscopic stoichiometry has been deteminced using RBS

Dramatically different defect pallens maty somelimes te found in widely separaled regins an a single waller. This suggests that the initiation of defect growth is not random and may te the result of some specific stimulus. A typical AFM imate of the comer of a highly defecked film is shown in figure 4 . In this image we sec a series nf micron-sized. rectangular asperitic: aligned with the underlying axes of the (2212) film. There are also some roughly circular asperities seen as well. The height of the rectangular defects is $l(X) \mathrm{nm}$ and of the circular defects is $20 \mathrm{~mm}$. These features are spaced at separation of about 5 microm. In between the asperities are flat regions similar to those imaged in figure 3. Il appears that these defects rise up from an otheruise smonth and well ordered film.

The teatures seen in the defect distribution in figure 4 are typical of those from all the wafers we have scanned. The lateral scale of the features is typically one micron or less. There have been occasional defects larser uhan a square micron in area. The variation in height and shape can he more extreme. One film had asperities extending as much as 1000 nm above the film surtace along with defects of only a few mm higin. While the area of the defects is unifonm. the height and shape of the asperities can vary greaty. The regions between defect structures are smondh all the BSCCO unit cell levicl.

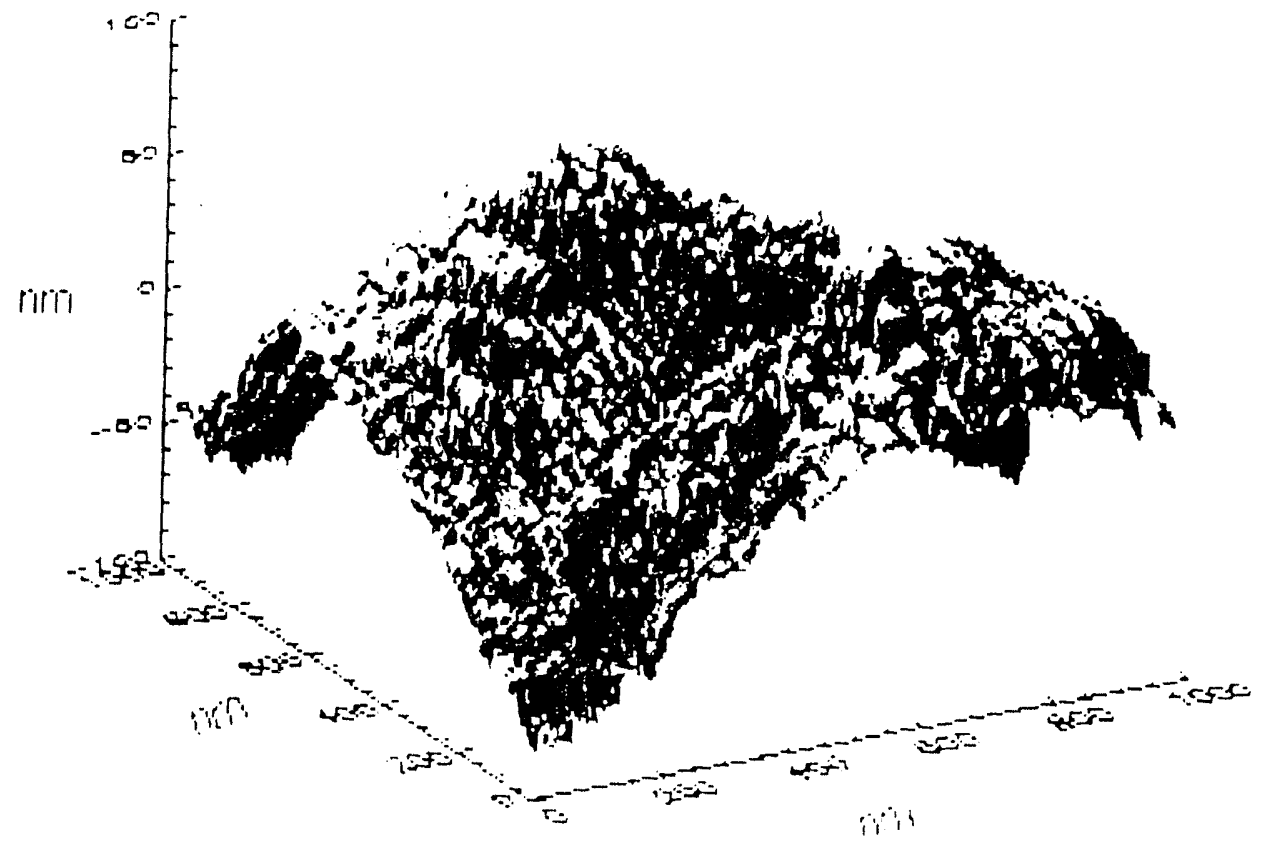

Iigure:

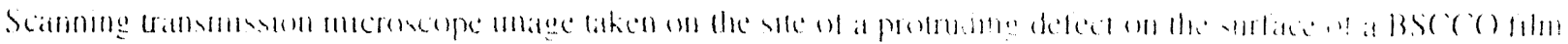

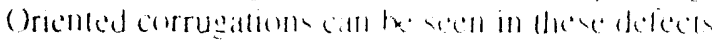


Images taken with the AIM only show the shape of the surface features and leave several important questions unresolved For instance, the defects may be precipitate phases segregated from the film in order to preserve the overall stoichiometry. In this case the chemical composition and electrical transport of the defects may be different from the surrounding (2212) film. SIM probing of the defects can in principal determine the resistivity of the defects and highlight differences between the defects and the rest of the film. Figure 5 shows an STM image of a defect similar to that imaged by AFM in figure 4 . Preliminary I-V characteristics taken in air showed that in general the defects were more conductive than the flat areas of the film. However possible surface contamination prevents more definitive conclusions. The structure of the defect imaged in figure 5 is typical of the asperities which are laterally oriented with respect to the (2212) film. There are cornugations on the surface of the defect suggestive of steps or layers running perpendicular to the film surface and over the top of the defect. This corrugation is not seen in the film surface and is evidence that the defect is not simply a cluster of BSCCO material. The defects pictured in figure 5 appear to have a crystal structure with a strong symmetry plane in the vertical direction. STM results have only been obtained for oriented defects of this type. Other types of defects may show different structure and electrical properties.

To obtain information about the chemical structure and the thickness of the surface asperities, we performed both SIMS and RBS over areas of low and high defect concentration. A Cameca IMS $3 f$ ion microscope was used to obtain the SIMS images. With this ion microscope, images can be formed using the secondary ion flux with lateral spatial resolution as fine as one micron. Depth profiles which track all of the major constituents and several suspected contaminates can be performed on 25 micron-wide areas.

The ion microscope was operated in two data collection modes. First ion fluxes of the expected elemental constituents were obtained in several widely separated spots on the film in order to search for local deviations from the expected stoichiometry. Scratches resulting from handling were clearly seen as regions of low copper and bismuth concentration and high strontium and titanium concentrations. Overlaying particles of foreign material were observed as a low concentration of all of the film constituents and a high concentration of unrelated elements such as sodium. Although these extraneous features could be resolved, no strong deviations from the 2212 stoichiometry were observed in these images. Large local stoichiometry variations would be expected if the asperities were precipitates of a second phase. The second SIMS imaging mode is to scan the probing ion beam in order to obtain a separate depth-dependent image profile for each of the BSCCO constituents. Defects that were significantly thicker than the film or those having a different ion sputtering rate would clearly show up in these scans. In spite of the high defect density in the region chosen for these measurements, there was no statistically significant feature seen that could be interpreted as a locally non-stoichiometric asperity. Therefore, one can conclude that the asperities have a stoichiometry similar to the (2212) film.

The oriented defects are usually confined to one corner of the wafers, which often have low defect concentrations overall. Both microscopic elemental analysis using the ion microscope and macroscopic analysis using RBS were performed at several locations on these wafers, including the high-defect comer and the center region which is nearly defect-free. Significant variations in the elemental concentrations were found in RBS and reproduced in the ion microscope. The strong correspondence of the microscopic and macroscopic measurements suggests that elemental concentrations are varying on a length scale of millimeters rather than microns. In the RB: data shown in tab?e 1 , both changes in the overall sample thickness and the locally determined stoichiometry were seen for copper and bismuth Statistically useful results were not obtained for calcium, strontium and oxygen in the film as their peaks were obscured by scattering from the strontium titanate substrate. The $10 \%$ variation in the elemental concentrations in this film is large compared to other films with fewer defects. Particle-induced $x$-ray emission (PIXE) spectra were also taken on these same films for comparison to the RBS and SIMS. This particular sample contained films of (2201) and (2212). The expected elemental concentrations are given in table 1. The measured elemental concentrations were nominally as expected for bismuth and copper-rich by all three techniques. In spite of being copper-rich, large areas of the sample were flat like the area shown in Figure 3 . 


\begin{tabular}{|c|l|c|c|}
\hline \hline Position & \multicolumn{1}{|c|}{ Defect Type } & $\begin{array}{c}\text { Cu concentration } \\
\left(10^{16} \mathrm{~cm}^{-2}\right)\end{array}$ & $\begin{array}{c}\text { Bi concentration } \\
\left(10^{16} \mathrm{~cm}^{-2}\right)\end{array}$ \\
\hline Corner & High-density, oriented & 6.10 & 6.12 \\
\hline Center & Low-density, not oriented & 6.10 & 5.53 \\
\hline Off-center & Low-density, not oriented & 6.10 & 5.98 \\
\hline \hline Calculated & None & & 5.79 \\
\hline
\end{tabular}

Table 1

Elemental concentrations determined by RBS for a film nominally composed of 10 layers of (2201) and 32 layers of (2212). Data are shown for both defected and defect-free regions.

\section{DISCUSSION}

Epitaxial thin films grown by the RCE technique are fabricated with precise control over the layering and composition. TEM shows that the bismuth-oxygen planes are coherent over distances of $100 \mathrm{~nm}$. At the interface with the substrate, this long coherence length allows the first (2212) layers to conceal the small-scale surface roughness of the substrate. The factors controlling the lateral coherence length of the (2212) film have not yet been identified. Two considerations may play a major role: control of the stoichiometry and the kinetics of the chemical assembly during deposition. The in-plane and c-axis diffusion constants are determined by the surface potential that a newly deposited atom sees. For instance, these potentials control whether growth occurs by cluster formation or layer-by-layer. Some control over the kinetics of growth can be accomplished by variation of the deposition temperature and elemental flux rates. Real-time RHEED observations show that Bi assembles in single layers, while $\mathrm{Ca}$ deposition forms clusters which then reconstruct upon the addition of the Cu overlayer. This reconstruction can be avoided by simultaneous deposition of the $\mathrm{Ca}$ and $\mathrm{Cu}$ overlayer. The fact that the first few (2212) layers are typically flatter than the substrate surface suggests that the kinetic factors strongly support the formation of continuous, homogenous layers.

The second important factor is control of the stoichiometry during deposition. According to RBS measurements, stoichiometry is presently controlled to within about $2 \%$. However, larger inhomogeneities in composition can appear in the films if the substrate temperature is non-uniform. We have seen large variations on the $\mathrm{mm}$ scale of elemental concentrations in films that have a low concentration of micron-scale defects on the film surface. Some of the variation from perfect stoichiometry may alternately be accommodated by the more extended imperfections observed in TEM, such as incomplete layers of the superconducting phases.

Larger asperities are also observed the surfaces of these heterostructures. Some of these asperities rise above the film surface as much as 1 micron, but they are more typically 10 to $100 \mathrm{~nm}$ high. Areal dimensions of one square micron are most common, but appear to be an upper bound. The nucleation source of these defects remains undetermined. The defects measured so far have chemical composition similar to the surrounding film. Some of the asperities have corrugated surfaces, with layer 
planes apparently growing up perpendicular to the plane of the film. a-axis grow th may te the source of these features. Further measurements in progress are underway to confirm this hypothesis.

We can draw some preliminary conclusions from the variety of techniques we have used to characterize the BSCCO films and trilayers. First, control of both the stoichiometry and the kinetic variables of the deposition are important in producing thin films free from large inhomogeneities. The accommodation of excess copper into a smooth (2212) film described suggests that more than a stoichiometric imbalance is required for the growth of large defects. Perhaps there is a competition between the growth of additional BSCCO planes and the nucleation of three-dimensional, non-wetting asperities. The importance of nucleation processes in the growth of large defects is underscored by the high quality of most of the BSCCO material. The substrate smoothness and strong tendency of the system to assemble in islands which encompass small irregularities seems to rule out substrate roughness as a dominant source of nucleation sites. However, substrate features such as scratches or dirt may serve as nucleation sites for the large defects. Several important issues remain, such as whether surface asperities extend deep into the (2212) films, and whether the crystalline orientation of defects varies from the nominal orientation. The formation of imperfections in the BSCCO system is unusual because of the anisotropic crystal structure and the many layered phases which can be formed. The insights gained from the study of BSCCO may help us understand the mechanisms of growth in other anisotropic crystals.

We thank Sandia National Laboratory and George Thomas for use of the TEM. Part of this work was performed under the auspices of the U.S. Department of Energy by Lawrence Livenmore National Laboratory under contract No. W7405-ENG-48.

\section{References}

1. J. N. Eckstein, I. Bozovic, D. G. Schlom, and J. S. Harris Jr., Appl. Phys. Lett. 57, 931 (1990)

2. J. N. Eckstein, I. Bozovic, D. G. Schlom, and J. S. Harris Jr., Appl. Phys. Lett. 57, 1049 (1990).

3. I. Bozovic, J. N. Eckstein, M. E. Klausmeier-Brown, and G. F. Virshup, Joumal of Superconductivity 5, 19 (1992).

4. M. E. Klausmeier-Brown, J. N. Eckstein, I. Bozovic, and G. F. Virshup, Appl. Phys. Lett 60, 657 (1992).

5. J. N. Eckstein, I. Bozovic, M. E. Klausmeier-Brown, G. Virshup, and K. S. Ralls, Materials Research Society Bulletin 17, 27 (1992).

6. G. F. Virshup, M. E. Klausmeier-Brown, J. N. Eckstein, and I. Bozovic, Appl. Phys. Lett. 60, 2288 (1992).

7. M. E. Klausmeier-Brown, G. F. Virshup, I. Bozovic, J. N. Eckstein, and K. S. Ralls, Appl. Phys. Lell. 61, 2806 (1992).

8. I. Bozovic, J. N. Eckstein, G. H. Virshup, A. Chaiken, M. Wall, R. Howell, and M. Fluss, J. Superconducuvity (1993) in press. 

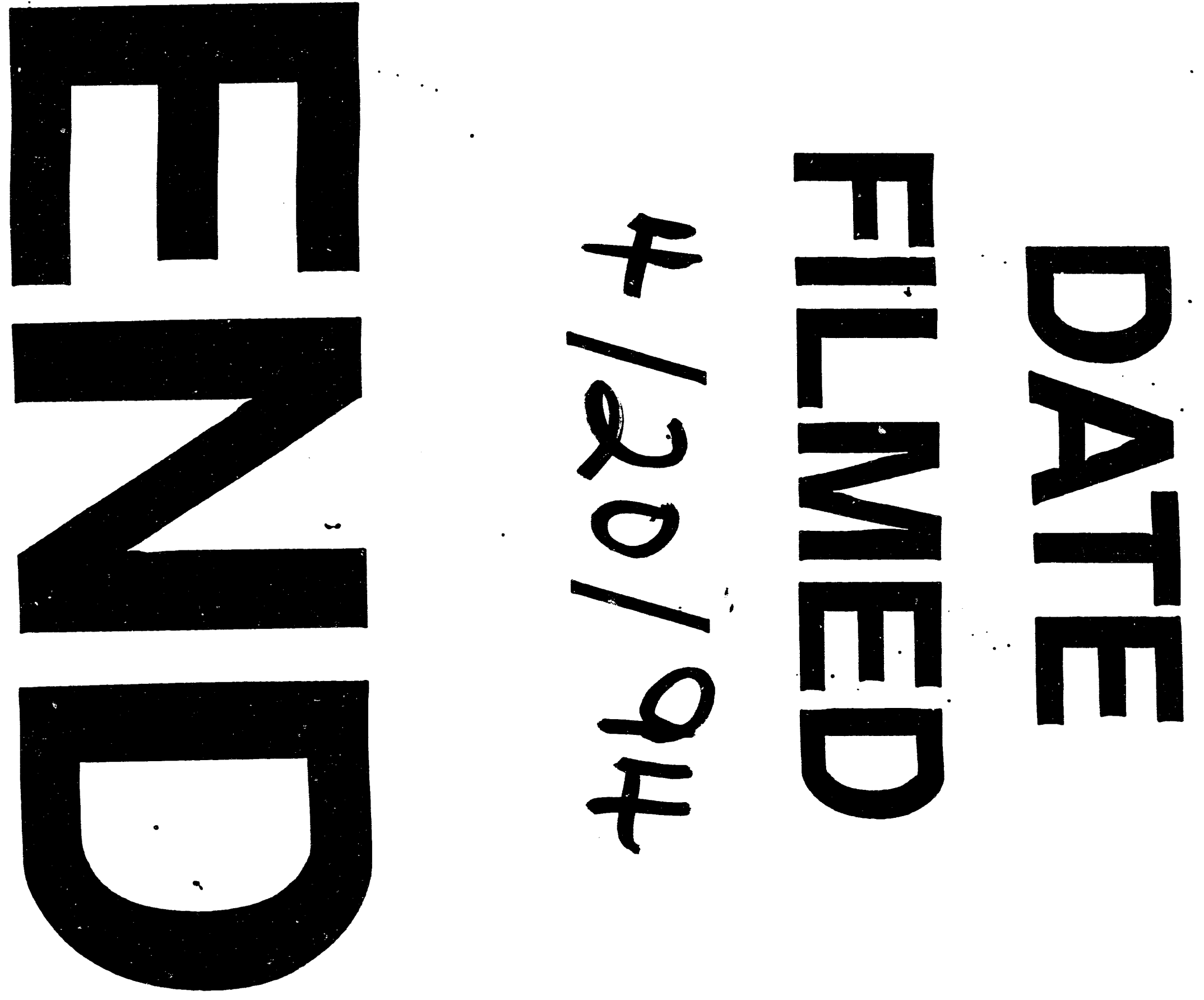
